\title{
ON THE RELATIONS BETWEEN CHARACTERISTIC CLASSES OF STABLE BUNDLES OF RANK 2 OVER AN ALGEBRAIC CURVE
}

\author{
BY P. E. NEWSTEAD
}

\begin{abstract}
We describe a complete set of generators and relations for a certain quotient of the rational cohomology ring of the moduli space of stable bundles of rank 2 and fixed determinant of odd degree over a nonsingular complex algebraic curve. The formulae for the relations apply in any genus and are relatively simple.
\end{abstract}

1. Introduction. Let $S=U_{L}(2,1)$ denote the moduli space of stable bundles of rank 2 and determinant $L$ of degree 1 over a nonsingular complete algebraic curve $X$ of genus $g \geq 2$ defined over the complex numbers. The Betti numbers of $S$ were determined some time ago in [3], and generators for $H^{*}(S ; Q)$ were given in [4]. Recently there has been renewed interest in obtaining a complete description of $H^{*}(S ; Q)$, particularly in connection with the work of M. F. Atiyah and R. Bott $[1, \S 9]$. David Mumford and Dave Bayer have performed some calculations on a computer, which provide evidence in support of some conjectures of Mumford. In this note we use the topological methods of $[3,4]$ to obtain some information about relations in $H^{*}(S ; Q)$; these provide further support for Mumford's conjectures.

2. The main theorem. We recall the generators for $H^{*}(S ; Q)$ given in [4], namely $\alpha \in H^{2}(S ; Z) ; \psi_{1}, \ldots, \psi_{2 g} \in H^{3}(S ; Z) ; \beta \in H^{4}(S ; Z)$. A little care is needed over the definition of the $\psi_{i}$. We first choose a symplectic basis $a_{1}, \ldots, a_{2 g}$ for $H^{1}(X ; Z)$ (with respect to the skew-symmetric form given by Poincaré duality); then the $\psi_{i}$ are defined by the equation

$$
\psi=\psi_{1} \otimes a_{1}+\cdots+\psi_{2 g} \otimes a_{2 g},
$$

where $\psi$ is the component in $H^{3}(S ; Z) \otimes H^{1}(X ; Z)$ of the second Chern class of a universal bundle on $S \times X$. We write

$$
\sigma=\psi_{1} \psi_{2}+\cdots+\psi_{2 g-1} \psi_{2 g} \in H^{6}(X ; Z)
$$

so that $\psi^{2}[X]=2 \sigma$.

TheOREM 1. Let $A$ denote the ring $H^{*}(S ; Q) /\langle\beta\rangle$. Then the monomials

$$
\alpha^{s} \psi_{q_{1}} \cdots \psi_{q_{t}} \quad\left(s, t \geq 0,1 \leq q_{1}<q_{2}<\cdots<q_{t} \leq 2 g, s+t<g\right)
$$

form a basis for $A$ as a vector space over $Q$. Moroever, whenever $s+t \geq g$,

$$
\left[\alpha^{s}+f_{s}(\alpha, \sigma)\right] \psi_{q_{1}} \cdots \psi_{q_{t}}=0,
$$

Received by the editors September 6, 1983.

1980 Mathematics Subject Classification. Primary 14D22, 14F05, 14F25; Secondary 55R40.

(c) 1984 American Mathematical Society $0273-0979 / 84 \$ 1.00+\$ .25$ per page 
where

$$
\sum_{u \geq 1}\left(\begin{array}{c}
s \\
3 u
\end{array}\right)\left(\begin{array}{c}
3 u-1 \\
2
\end{array}\right)\left(\begin{array}{c}
3 u-4 \\
2
\end{array}\right) \cdots\left(\begin{array}{l}
2 \\
2
\end{array}\right) \alpha^{s-3 u}(8 \sigma)^{u} .
$$

Note that (2) can be used to express every element of $A$ as a linear combination of the basis elements (1); hence (1) is a complete set of relations for $A$ as a graded $Q$-algebra. The elements $\alpha, \beta$ and $\sigma$ correspond to those denoted by $h, h^{2}-4 \nu$ and $-\theta$ in $[5, \S 5]$. It will be noted that our result, though it does not give a complete description of $H^{*}(S ; Q)$, is a precise one; we hope that it will be of help in obtaining a complete result. Note further that $f_{s}(\alpha, \sigma)$ is independent of $g$; this provides partial verification of one of Mumford's conjectures.

3. Subsidiary results. We recall from $[3,4]$ the subspaces $S_{0}^{(g)}$ and $N^{(g)^{\prime}}$ of $S U(2)^{2 g}$ defined by

$$
\begin{gathered}
\left(A_{1}, \ldots, A_{2 g}\right) \in S_{0}^{(g)} \Leftrightarrow\left(A_{1} A_{2} A_{1}^{-1} A_{2}^{1}\right) \cdots\left(A_{2 g-1} A_{2 g} A_{2 g-1}^{-1} A_{2 g}^{-1}\right)=-I, \\
\left(A_{1}, \ldots, A_{2 g}\right) \in N^{(g)^{\prime}} \Leftrightarrow \operatorname{Trace}\left[\left(A_{1} A_{2} A_{1}^{-1} A_{2}^{1}\right) \cdots\left(A_{2 g-1} A_{2 g} A_{2 g-1}^{-1} A_{2 g}^{-1}\right)\right] \geq 0 .
\end{gathered}
$$

With the notation of $[4, \S 3]$, and writing $\sigma=\mu_{1} \mu_{2}+\cdots+\mu_{2 g-1} \mu_{2 g}$, we shall prove

THEOREM 2. For $r \leq 3 g-3$, the monomials

$$
\lambda^{s} \mu_{q_{1}} \cdots \mu_{q_{t}} \quad\left(s, t \geq 0,1 \leq q_{1}<q_{2}<\cdots<q_{t} \leq 2 g, 2 s+3 t=r\right),
$$

with $s+t \leq g-1$, form a basis for $H^{r}\left(S_{0}^{(g)} ; Q\right)$. Moreover, whenever $s+t \geq g$,

$$
\left[\lambda^{s}+g_{s}(\lambda, \sigma)\right] \mu_{q_{1}} \cdots \mu_{q_{t}}=0,
$$

where

$$
g_{s}(\lambda, \sigma)=f_{s}(\lambda, k \sigma / 8)
$$

and $k$ is a constant independent of $g$.

THEOREM 3. For $r \leq 3 g$, the monomials (3) with $s+t \leq g$ form a basis of $H^{r}\left(N^{(g)^{\prime}} ; Q\right)$. Moreover, (4) holds whenever $s+t \geq g+1$.

4. Outline of proofs. Theorem 1 can be deduced from Theorem 2 by recalling [2, 3] that there is a principal $P U(2)$-fibration $p: S_{0}^{(g)} \rightarrow S$. Moreover, by $[4$, Proposition 2.6$]$, the ideal $\langle\beta\rangle$ in $H^{*}(S ; Q)$ coincides with the kernel of $p^{*}$, and it follows from the formulae for the Betti numbers in [3] that $p^{*}$ is zero above degree $3 g-3$. One can check also that $p^{*}\left(\psi_{i}\right)=\mu_{i}$ : while $p^{*}(\alpha)$ is certainly a multiple of $\lambda$. So Theorem 2 implies a modified version of Theorem 1 in which (2) is replaced by $\left[\alpha^{s}+f_{s}\left(\alpha, k^{\prime} \sigma\right)\right] \psi_{q_{1}} \cdots \psi_{q_{t}}=0$, where $k^{\prime}$ is a constant which could possibly depend on $g$. It remains to prove that $k^{\prime}=1$, which we do by using the family of stable bundles constructed by Ramanan in [5, §4] and making some explicit computations.

The first assertion of Theorem 2 follows from [4, Proposition 3.4] by using the formulae of [3, Theorem 2]. The relations [4] follow from those of Theorem 3 by using the maps

$$
h: N^{(g-1)^{\prime}} \rightarrow S_{0}^{(g)} \quad(\text { see }[4, \text { p. 341] }),
$$




$$
\rho: S_{0}^{(g)} \rightarrow S_{0}^{(g)}: \rho\left(A_{1}, \ldots, A_{2 g}\right)=\left(A_{2 g-1}, A_{2 g}, A_{1}, \ldots, A_{2 g-2}\right) .
$$

Let $\delta$ be an element of $H^{r}\left(S_{0}^{(g)} ; Q\right)$ with $r \leq 3 g-3$; by writing $\delta$ as a linear combination of (3) and making some direct computations, we see that

$$
\delta=0 \Leftrightarrow\left(\rho^{i} \circ h\right)^{*}(\delta)=0 \text { for } 0 \leq i \leq g-1 .
$$

A further direct computation proves (4).

Finally, the first assertion of Theorem 3 follows from [4, Proposition 3.3]. For the relations, we argue by induction on $g$, using the maps

$$
l: N^{(g-1)^{\prime}} \times N^{(1)^{\prime}} \rightarrow N^{(g)^{\prime}}, \quad m: N^{(g-2)^{\prime}} \times N^{(2)^{\prime}} \rightarrow N^{(g)^{\prime}}
$$

defined in $[4$, p. $342 ; 3$, p. 256], and an assertion similar to (5) with respect to these maps. The cases $g=1$ and $g=2$ must be checked separately. (For $g=2$ we get just one relation $\lambda^{3}+k \sigma=0$; this is the source of the constant k.)

Further details of the proofs, and some related results, will appear elsewhere.

\section{ReferenCes}

1. M. F. Atiyah and R. Bott, The Yang-Mills equations over Riemann surfaces, Philos. Trans. Roy. Soc. London Ser. A 308 (1982), 523-615.

2. M. S. Narasimhan and C. S. Seshadri, Stable and unitary vector bundles on a compact Riemann surface, Ann. of Math. (2) 82 (1965), 540-567.

3. P. E. Newstead, Topological properties of some spaces of stable bundles, Topology 6 (1967), 241-262.

4. - Characteristic classes of stable bundles of rank 2 over an algebraic curve, Trans. Amer. Math. Soc. 169 (1972), 337-345.

5. S. Ramanan, The moduli spaces of vector bundles over an algebraic curve, Math. Ann. 200 (1973), 69-84.

Department of Pure Mathematics, The University, Liverpool L69 3BX, ENGLAND 